\title{
Coefficient bounds for a subclass of Sakaguchi type functions using Chebyshev Polynomial
}

\author{
N.P. Damodaran ${ }^{1}$, Srutha Keerthi ${ }^{2}$ \\ ${ }^{1}$ Research Scholar, Mathematics Division School of Advanced Sciences, VIT Chennai \\ ${ }^{2}$ Mathematics Division School of Advanced Sciences, VIT Chennai
}

\section{Article Info}

Article history:

Received Jan $12^{\text {th }}, 2018$

Revised May $20^{\text {th }}, 2018$

Accepted Jun $26^{\text {th }}, 2018$

\author{
ABSTRACT \\ In this work, considering a general subclass of bi-univalent \\ Sakaguchi type functions and using Chebyshev polynomials, we \\ obtain coefficient expansions for functions in this class.
}

\section{Keyword:}

bi-univalent functions coefficient estimates Chebyshev polynomials Sakaguchi type functions subordination.

\section{Corresponding Author:}

\section{Srutha Keerthi,}

Mathematics Division

School of Advanced Sciences

VIT Chennai, Vandaloor, Kelambakkam Road

Chennai - 600 127, India

Email:sruthilaya06@yahoo.co.in

\section{Introduction}

Let $\mathrm{D}$ be the unit disk $\{\mathrm{z}: \mathrm{z} \in \mathrm{C}$ and $|\mathrm{z}|<1\}$, A be the class of all functions analytic in $\mathrm{D}$, satisfying the conditions

$$
f(0)=0 \text { and } f^{\prime}(0)=1
$$

Then each function $\mathrm{f}$ in $\mathrm{A}$ has the Taylor expansion

$$
f(z)=z+\sum_{n=2}^{\infty} a_{n} z^{n}
$$


Further, by $\mathrm{S}$ we shall denote the class of all functions in A which are univalent in D. The koebe one-quarter theorem [7] states that the image of D under every function $\mathrm{f}$ from $\mathrm{S}$ contains a disk of radius $\frac{1}{4}$, Thus every such univalent function has an inverse $\mathrm{f}^{-1}$ which satisfies

$\mathrm{f}^{-1}(\mathrm{f}(\mathrm{z}))=\mathrm{z}, \quad(\mathrm{z} \in \mathrm{D})$

and

$$
\mathrm{f}\left(\mathrm{f}^{-1}(\mathrm{w})\right)=\mathrm{w}, \quad\left(|\mathrm{w}|<\mathrm{r}_{0}(\mathrm{f}) ; \mathrm{r}_{0}(\mathrm{f}) \geq \frac{1}{4}\right)
$$

where

$$
\mathrm{f}^{-1}(\mathrm{w})=\mathrm{w}-\mathrm{a}_{2} \mathrm{w}^{2}+\left(2 \mathrm{a}_{2}^{2}-\mathrm{a}_{3}\right) \mathrm{w}^{3}-\left(5 \mathrm{a}_{2}^{3}-5 \mathrm{a}_{2} \mathrm{a}_{3}+\mathrm{a}_{4}\right) \mathrm{w}^{4}+\cdots
$$

A function $\mathrm{f}(\mathrm{z}) \in \mathrm{A}$ is said to be bi-univalent in $\mathrm{D}$ if both $\mathrm{f}(\mathrm{z})$ and $\mathrm{f}^{-1}(\mathrm{z})$ are univalent in $\mathrm{D}$. If the functions $f$ and $g$ are analytic in $D$, then $f$ is said to be subordinate to $g$, written as

$\mathrm{f}(\mathrm{z}) \prec \mathrm{g}(\mathrm{z}), \quad(\mathrm{z} \in \mathrm{D})$

if there exists a Schwarz function w(z), analytic in D, with

$\mathrm{w}(0)=0 \quad$ and $\quad|\mathrm{w}(\mathrm{z})|<1 \quad(\mathrm{z} \in \mathrm{D})$

such that $f(z)=g(w(z)) \quad(z \in D)$.

Let $\Sigma$ denote the class of bi-univalent functions defined in the unit disk D. Lewin [11] studied the class of bi-univalent functions, obtaining the bound 1.51 for modulus of the second coefficients $\left|\mathrm{a}_{2}\right|$. Netanyahu [14] showed that $\max \left|\mathrm{a}_{2}\right|=\frac{4}{3}$ if $\mathrm{f}(\mathrm{z}) \in \Sigma$. Subsequently, Brannan and Clunie [4] conjectured that $\left|\mathrm{a}_{2}\right| \leq \sqrt{2}$ for $\mathrm{f} \in \Sigma$. Brannan and Taha [3] introduced certain subclasses of the bi-univalent function class $\Sigma$ similar to find familiar subclasses. Recently, many authors investigated bounds for various subclasses of biunivalent functions ([1], [6], [12], [15], [17]).

Not much is known about the bounds on the general coefficient $\left|a_{n}\right|$ for $n \geq 4$. In the literature, there are only a few works determining the general coefficient bounds $\left|a_{n}\right|$ for the analytic bi-univalent functions ([2], [5], [9], [10]). The coefficient estimate problem for each of $\left|a_{n}\right|(n \in N /\{1,2\} ; N=\{1,2,3, \ldots\})$ is still an open problem.

Chebyshev polynomials have become increasingly important in numerical analysis, from both theoretical and practical points of view. There are four kinds of Chebyshev polynomials. The majority of books and research papers dealing with specific orthogonal polynomials of Chebyshev family, contain mainly results of Chebyshev polynomials of first and second kinds $\mathrm{T}_{\mathrm{n}}(\mathrm{x})$ and $\mathrm{U}_{\mathrm{n}}(\mathrm{x})$ and their numerous uses in different applications, see for example, Doha [8] and Mason [13].

The Chebyshev polynomials of the first and second kinds are well known. In the case of a real variable $\mathrm{x}$ on $[-1,1]$, they are defined by

$$
\mathrm{T}_{\mathrm{n}}(\mathrm{x})=\cos \mathrm{n} \theta,
$$




$$
\mathrm{U}_{\mathrm{n}}(\mathrm{x})=\frac{\sin (\mathrm{n}+1) \theta}{\sin \theta}
$$

where the subscript $\mathrm{n}$ denotes the polynomial degree and where $\mathrm{x}=\cos \theta$.

\section{Definition 1}

For $0 \leq \lambda \leq 1, l \in\left(\frac{1}{2}, 1\right],|\mathrm{t}| \leq 1$ and $\mathrm{t} \neq 1$ ( $\mathrm{t}$ is real), a function $\mathrm{f}(\mathrm{z}) \in \Sigma$ is said to be in the class $\mathrm{C}_{\Sigma}(\lambda$, $l, \mathrm{t})$, if the following subordination hold

$$
\left\{\begin{array}{c}
\frac{(1-\mathrm{t})\left[\mathrm{zf}^{\prime}(\mathrm{z})+\left(2 \lambda^{2}-\lambda\right) \mathrm{z}^{2} \mathrm{f}^{\prime \prime}(\mathrm{z})\right]}{\left[4\left(\lambda-\lambda^{2}\right)(\mathrm{z}-\mathrm{tz})+\left(2 \lambda^{2}-\lambda\right) z\left(\mathrm{f}^{\prime}(\mathrm{z})-t \mathrm{f}^{\prime}(\mathrm{tz})\right)\right.} \\
\left.+\left(2 \lambda^{2}-3 \lambda+1\right)(\mathrm{f}(\mathrm{z})-\mathrm{f}(\mathrm{tz}))\right]
\end{array}\right\} \prec H(\mathrm{z}, l)=\frac{1}{1-2 l \mathrm{z}+\mathrm{z}^{2}} \quad(\mathrm{z} \in \mathrm{D})
$$

and

$$
\left\{\begin{array}{c}
\frac{(1-\mathrm{t})\left[\mathrm{wg}^{\prime}(\mathrm{w})+\left(2 \lambda^{2}-\lambda\right) w^{2} \mathrm{~g}^{\prime \prime}(\mathrm{w})\right]}{\left[4\left(\lambda-\lambda^{2}\right)(\mathrm{w}-\mathrm{tw})+\left(2 \lambda^{2}-\lambda\right) w\left(\mathrm{~g}^{\prime}(\mathrm{w})-t \mathrm{~g}^{\prime}(\mathrm{tw})\right)\right.} \\
\left.+\left(2 \lambda^{2}-3 \lambda+1\right)(g(\mathrm{w})-g(\mathrm{tw}))\right]
\end{array}\right\} \prec H(\mathrm{w}, l)=\frac{1}{1-2 l \mathrm{w}+\mathrm{w}^{2}} \quad(\mathrm{w} \in \mathrm{D})
$$

where $g(w)=f^{-1}(w)$

$$
\text { We note that if } l=\cos \alpha, \alpha \in\left(\frac{-\pi}{3}, \frac{\pi}{3}\right) \text {, then }
$$

$\mathrm{H}(\mathrm{z}, l)=\frac{1}{1-2 l \mathrm{z}+\mathrm{z}^{2}}=1+\sum_{\mathrm{n}=1}^{\infty} \frac{\sin (\mathrm{n}+1) \alpha}{\sin \alpha} \mathrm{z}^{\mathrm{n}} \quad(\mathrm{z} \in \mathrm{D})$.

Thus

$\mathrm{H}(\mathrm{z}, l)=1+2 \cos \alpha \mathrm{z}+\left(3 \cos ^{2} \alpha-\sin ^{2} \alpha\right) \mathrm{z}^{2}+\cdots \quad(\mathrm{z} \in \mathrm{D})$.

Following see, we write

$$
\mathrm{H}(\mathrm{z}, l)=1+\mathrm{U}_{1}(l) \mathrm{z}+\mathrm{U}_{2}(l) \mathrm{z}^{2}+\cdots \quad(\mathrm{z} \in \mathrm{D}, l \in(-1,1)),
$$

where

$$
\mathrm{U}_{\mathrm{n}-1}=\frac{\sin (\operatorname{narccos} l)}{\sqrt{1-l^{2}}}
$$

$(\mathrm{n} \in \mathrm{N})$ are the chebyshev polynomials of the second kind. Also it is known that 
$\mathrm{U}_{\mathrm{n}}(l)=2 l \mathrm{U}_{\mathrm{n}-1}(l)-\mathrm{U}_{\mathrm{n}-2}(l)$,

and

$$
\mathrm{U}_{1}(l)=2 l, \quad \mathrm{U}_{2}(l)=4 l^{2}-1, \quad \mathrm{U}_{3}(l)=8 l^{3}-4 l, \ldots
$$

The Chebyshev polynomials $\mathrm{T}_{\mathrm{n}}(l), l \in[-1,1]$, of the first kind have the generating function of the form $\sum_{\mathrm{n}=0}^{\infty} \mathrm{T}_{\mathrm{n}}(l) \mathrm{z}^{\mathrm{n}}=\frac{1-l \mathrm{z}}{1-2 l \mathrm{z}+\mathrm{z}^{2}} \quad(\mathrm{z} \in \mathrm{D})$.

However, the Chebyshev polynomials of the first kind $\mathrm{T}_{\mathrm{n}}(l)$ and the second kind $\mathrm{U}_{\mathrm{n}}(l)$ are well connected by the following relationships

$$
\begin{array}{r}
\frac{\mathrm{dT}_{\mathrm{n}}(l)}{\mathrm{d} l}=\mathrm{nU}_{\mathrm{n}-1}(l), \\
\mathrm{T}_{\mathrm{n}}(l)=\mathrm{U}_{\mathrm{n}}(l)-l \mathrm{U}_{\mathrm{n}-1}(l), \\
2 \mathrm{~T}_{\mathrm{n}}(l)=\mathrm{U}_{\mathrm{n}}(l)-\mathrm{U}_{\mathrm{n}-2}(l) .
\end{array}
$$

In this paper, motivated by the earlier work of Srutha keerthi [16].

\section{Coefficient bounds for the function class $\mathbf{C}_{\Sigma}(\lambda, l, t)$}

\section{Theorem 1}

Let the function $\mathrm{f}(\mathrm{z})$ given by $(1.1)$ be in the class $\mathrm{C}_{\Sigma}(\lambda, l, \mathrm{t})$. Then

$$
\begin{aligned}
& \left|\mathrm{a}_{2}\right| \leq \frac{2 l \sqrt{2 l}\left(2 \lambda^{2} \mathrm{t}-\lambda \mathrm{t}+1\right)}{\sqrt{|\mathrm{A}|}}, \\
& \left|\mathrm{a}_{3}\right| \leq \frac{4 l^{2}\left(2 \lambda^{2} \mathrm{t}-\lambda \mathrm{t}+1\right)^{2}}{\left[2-\mathrm{u}_{2}\left(\left(2 \lambda^{2}-3 \lambda+1\right)+2 t\left(2 \lambda^{2}-\lambda\right)\right)\right]^{2}}+\frac{2 l\left(2 \lambda^{2} \mathrm{t}-\lambda \mathrm{t}+1\right)}{B}, \\
& \mathrm{~A}=4 l^{2} \mathrm{~B}\left(2 \lambda^{2} \mathrm{t}-\lambda \mathrm{t}+1\right)-\left\{\begin{array}{l}
\left(2-\mathrm{u}_{2}\left[\left(2 \lambda^{2}-3 \lambda+1\right)+2 t\left(2 \lambda^{2}-\lambda\right)\right]\right) \\
{\left[4 l^{2}\left(4 \lambda^{2}-2 \lambda+2\right)+\left(2-\mathrm{u}_{2}\left[\left(2 \lambda^{2}-3 \lambda+1\right)+2 t\left(2 \lambda^{2}-\lambda\right)\right]\right)\right]}
\end{array}\right\}, \\
& B=\left[\left(6 \lambda^{2}-3 \lambda+3\right)-\mathrm{u}_{3}\left[\left(2 \lambda^{2}-3 \lambda+1\right)+3 \mathrm{t}\left(2 \lambda^{2}-\lambda\right)\right]\right] .
\end{aligned}
$$


and $\quad \mathrm{u}_{\mathrm{n}}=\frac{1-\mathrm{t}^{\mathrm{n}}}{1-\mathrm{t}}, \mathrm{n} \in \mathrm{N}$.

Proof.

Let $\mathrm{f} \in \mathrm{C}_{\Sigma}(\lambda, l, \mathrm{t})$. From (1.2) and (1.3), we have

$$
\begin{aligned}
& \left\{\begin{array}{c}
(1-\mathrm{t})\left[\mathrm{zf}^{\prime}(\mathrm{z})+\left(2 \lambda^{2}-\lambda\right) \mathrm{z}^{2} \mathrm{f}^{\prime \prime}(\mathrm{z})\right] \\
{\left[4\left(\lambda-\lambda^{2}\right)(\mathrm{z}-\mathrm{tz})+\left(2 \lambda^{2}-\lambda\right)\left(\mathrm{f}^{\prime}(\mathrm{z})-t \mathrm{f} \mathrm{f}^{\prime}(\mathrm{tz})\right)\right.} \\
\left.+\left(2 \lambda^{2}-3 \lambda+1\right)(\mathrm{f}(\mathrm{z})-\mathrm{f}(\mathrm{tz}))\right]
\end{array}\right\} \\
& =1+\mathrm{U}_{1}(l) \mathrm{w}(\mathrm{z})+\mathrm{U}_{2}(l) \mathrm{w}^{2}(\mathrm{z})+\cdots,
\end{aligned}
$$

and

$$
\begin{aligned}
& \left\{\begin{array}{c}
\frac{(1-\mathrm{t})\left[\mathrm{wg}^{\prime}(\mathrm{w})+\left(2 \lambda^{2}-\lambda\right) w^{2} \mathrm{~g}^{\prime \prime}(\mathrm{w})\right]}{\left[4\left(\lambda-\lambda^{2}\right)(\mathrm{w}-\mathrm{tw})+\left(2 \lambda^{2}-\lambda\right) w\left(\mathrm{~g}^{\prime}(\mathrm{w})-\mathrm{tg}^{\prime}(\mathrm{tw})\right)\right.} \\
\left.+\left(2 \lambda^{2}-3 \lambda+1\right)(g(\mathrm{w})-g(\mathrm{tw}))\right]
\end{array}\right\} \\
& =1+\mathrm{U}_{1}(l) \mathrm{v}(\mathrm{w})+\mathrm{U}_{2}(l) \mathrm{v}^{2}(\mathrm{w})+\cdots,
\end{aligned}
$$

for some analytic functions $\mathrm{w}, \mathrm{v}$ such that $\mathrm{w}(0)=\mathrm{v}(0)=0$ and $|\mathrm{w}(\mathrm{z})|<1,|\mathrm{v}(\mathrm{z})|<1$ for all $\mathrm{z} \in \mathrm{D}$. From the equalities (2.1) and (2.2), we obtain that

$$
\begin{gathered}
\left\{\begin{array}{c}
\frac{(1-\mathrm{t})\left[\mathrm{zf}^{\prime}(\mathrm{z})+\left(2 \lambda^{2}-\lambda\right) \mathrm{z}^{2} \mathrm{f}^{\prime \prime}(\mathrm{z})\right]}{\left[4\left(\lambda-\lambda^{2}\right)(\mathrm{z}-\mathrm{tz})+\left(2 \lambda^{2}-\lambda\right)\left(\mathrm{f}^{\prime}(\mathrm{z})-t \mathrm{f}^{\prime}(\mathrm{tz})\right)\right.} \\
\left.+\left(2 \lambda^{2}-3 \lambda+1\right)(\mathrm{f}(\mathrm{z})-\mathrm{f}(\mathrm{tz}))\right]
\end{array}\right\} \\
=1+\mathrm{U}_{1}(l) \mathrm{c}_{1} \mathrm{z}+\left[\mathrm{U}_{1}(l) \mathrm{c}_{2}+\mathrm{U}_{2}(l) \mathrm{c}_{1}^{2}\right] \mathrm{z}^{2}+\cdots
\end{gathered}
$$




$$
\begin{aligned}
& \left\{\begin{array}{c}
(1-\mathrm{t})\left[\mathrm{wg}^{\prime}(\mathrm{w})+\left(2 \lambda^{2}-\lambda\right) w^{2} \mathrm{~g}^{\prime \prime}(\mathrm{w})\right] \\
{\left[4\left(\lambda-\lambda^{2}\right)(\mathrm{w}-\mathrm{tw})+\left(2 \lambda^{2}-\lambda\right) w\left(\mathrm{~g}^{\prime}(\mathrm{w})-\mathrm{tg}^{\prime}(\mathrm{tw})\right)\right.} \\
\left.+\left(2 \lambda^{2}-3 \lambda+1\right)(g(\mathrm{w})-g(\mathrm{tw}))\right]
\end{array}\right\} \\
& =1+\mathrm{U}_{1}(l) \mathrm{d}_{1} \mathrm{w}+\left[\mathrm{U}_{1}(l) \mathrm{d}_{2}+\mathrm{U}_{2}(l) \mathrm{d}_{1}^{2}\right] \mathrm{w}^{2}+\cdots .
\end{aligned}
$$

It is fairly well-known that if $|\mathrm{w}(\mathrm{z})|=\left|\mathrm{c}_{1} \mathrm{z}+\mathrm{c}_{2} \mathrm{z}^{2}+\mathrm{c}_{3} \mathrm{z}^{3}+\ldots\right|<1$ and $|\mathrm{v}(\mathrm{w})|=\left|\mathrm{d}_{1} \mathrm{w}+\mathrm{d}_{2} \mathrm{w}^{2}+\mathrm{d}_{3} \mathrm{w}^{3}+\ldots\right|<1, \mathrm{z}, \mathrm{w} \in \mathrm{D}$, then

$\left|\mathrm{c}_{\mathrm{j}}\right| \leq 1, \quad \forall \mathrm{j} \in \mathrm{N}$.

It follows from (2.3) and (2.4) that

$$
\begin{gathered}
\frac{\left[2-\mathrm{u}_{2}\left(\left(2 \lambda^{2}-3 \lambda+1\right)+2 t\left(2 \lambda^{2}-\lambda\right)\right)\right]}{\left(2 \lambda^{2} t-\lambda \mathrm{t}+1\right)} \mathrm{a}_{2}=\mathrm{U}_{1}(l) \mathrm{c}_{1}, \\
\frac{B \mathrm{a}_{3}}{\left(2 \lambda^{2} t-\lambda \mathrm{t}+1\right)}-\frac{C \mathrm{a}_{2}^{2}}{\left(2 \lambda^{2} t-\lambda \mathrm{t}+1\right)^{2}}=\mathrm{U}_{1}(l) \mathrm{c}_{2}+\mathrm{U}_{2}(l) \mathrm{c}_{1}^{2}, \\
-\frac{\left[2-\mathrm{u}_{2}\left(\left(2 \lambda^{2}-3 \lambda+1\right)+2 t\left(2 \lambda^{2}-\lambda\right)\right)\right]}{\left(2 \lambda^{2} t-\lambda \mathrm{t}+1\right)} \mathrm{a}_{2}=\mathrm{U}_{1}(l) \mathrm{d}_{1}, \\
\frac{B\left(2 \mathrm{a}_{2}^{2}-\mathrm{a}_{3}\right)}{\left(2 \lambda^{2} t-\lambda \mathrm{t}+1\right)}-\frac{C \mathrm{a}_{2}^{2}}{\left(2 \lambda^{2} t-\lambda \mathrm{t}+1\right)^{2}}=\mathrm{U}_{1}(l) \mathrm{d}_{2}+\mathrm{U}_{2}(l) \mathrm{d}_{1}^{2},
\end{gathered}
$$

Where

$$
C=\left\{\left(2-\mathrm{u}_{2}\left[\left(2 \lambda^{2}-3 \lambda+1\right)+2 t\left(2 \lambda^{2}-\lambda\right)\right]\right)\left[2\left(2 \lambda^{2}-\lambda\right)\left[1+t \mathrm{u}_{2}\right]+\mathrm{u}_{2}\left(2 \lambda^{2}-3 \lambda+1\right)\right]\right\} .
$$

From (2.5) and (2.7) we obtain

$$
\mathrm{c}_{1}=-\mathrm{d}_{1}
$$

and

$$
\frac{2\left[2-\mathrm{u}_{2}\left(\left(2 \lambda^{2}-3 \lambda+1\right)+2 t\left(2 \lambda^{2}-\lambda\right)\right)\right]^{2}}{\left(2 \lambda^{2} t-\lambda \mathrm{t}+1\right)^{2}} \mathrm{a}_{2}^{2}=\mathrm{U}_{1}^{2}(l)\left(\mathrm{c}_{1}^{2}+\mathrm{d}_{1}^{2}\right) .
$$


By adding (2.6) and (2.8), we get

$\frac{2 B \mathrm{a}_{2}^{2}}{\left(2 \lambda^{2} t-\lambda \mathrm{t}+1\right)}-\frac{2 C \mathrm{a}_{2}^{2}}{\left(2 \lambda^{2} t-\lambda \mathrm{t}+1\right)^{2}}=\mathrm{U}_{1}(l)\left(\mathrm{c}_{2}+\mathrm{d}_{2}\right)+\mathrm{U}_{2}(l)\left(\mathrm{c}_{1}^{2}+\mathrm{d}_{1}^{2}\right)$,

By using (2.10) inequality (2.11), we have

$$
\frac{2\left[\mathrm{~B}\left(2 \lambda^{2}-\lambda \mathrm{t}+1\right)-C\right] \mathrm{a}_{2}^{2}}{\left(2 \lambda^{2} t-\lambda \mathrm{t}+1\right)^{2}}-\frac{2 \mathrm{U}_{2}(l)\left[2-\mathrm{u}_{2}\left(\left(2 \lambda^{2}-3 \lambda+1\right)+2 t\left(2 \lambda^{2}-\lambda\right)\right)\right]^{2} \mathrm{a}_{2}^{2}}{\mathrm{U}_{1}^{2}(l)\left(2 \lambda^{2} t-\lambda \mathrm{t}+1\right)^{2}}=\mathrm{U}_{1}(l)\left(\mathrm{c}_{2}+\mathrm{d}_{2}\right) \text {. }
$$

From (1.4) and (2.12) we get

$$
\left|\mathrm{a}_{2}\right| \leq \frac{2 l \sqrt{2 l}\left(2 \lambda^{2} \mathrm{t}-\lambda \mathrm{t}+1\right)}{\sqrt{|\mathrm{A}|}}
$$

Next, in order find the bound on $\left|\mathrm{a}_{3}\right|$, by subtracting (2.8) from (2.6), we obtain

$$
\frac{2 B\left(\mathrm{a}_{3}-\mathrm{a}_{2}^{2}\right)}{\left(2 \lambda^{2} t-\lambda \mathrm{t}+1\right)}=\mathrm{U}_{1}(l)\left(\mathrm{c}_{2}-\mathrm{d}_{2}\right)+\mathrm{U}_{2}(l)\left(\mathrm{c}_{1}^{2}-\mathrm{d}_{1}^{2}\right) .
$$

Then, in view of (2.9) and (2.10), we have from (2.13)

$$
\mathrm{a}_{3}=\frac{\mathrm{U}_{1}^{2}(l)\left(\mathrm{c}_{1}^{2}+\mathrm{d}_{1}^{2}\right)\left(2 \lambda^{2} t-\lambda \mathrm{t}+1\right)^{2}}{2\left[2-\mathrm{u}_{2}\left(\left(2 \lambda^{2}-3 \lambda+1\right)+2 t\left(2 \lambda^{2}-\lambda\right)\right)\right]^{2}}+\frac{\mathrm{U}_{1}(l)\left(\mathrm{c}_{2}-\mathrm{d}_{2}\right)\left(2 \lambda^{2} t-\lambda \mathrm{t}+1\right)}{2 \mathrm{~B}} .
$$

Notice that (1.4), we get

$$
\left|\mathrm{a}_{3}\right| \leq \frac{4 l^{2}\left(2 \lambda^{2} \mathrm{t}-\lambda \mathrm{t}+1\right)^{2}}{\left[2-\mathrm{u}_{2}\left(\left(2 \lambda^{2}-3 \lambda+1\right)+2 t\left(2 \lambda^{2}-\lambda\right)\right)\right]^{2}}+\frac{2 l\left(2 \lambda^{2} \mathrm{t}-\lambda \mathrm{t}+1\right)}{B} .
$$

$\square$

\section{Fekete-Szegö inequalities for the function class $\mathrm{C}_{\Sigma}(\lambda, l, t)$}

\section{Theorem 2}

Let $\mathrm{f}$ given by (1.1) be in the class $\mathrm{C}_{\Sigma}(\lambda, l, \mathrm{t})$ and $\mu \in \mathrm{R}$. Then 


$$
\left|\mathrm{a}_{3}-\mu \mathrm{a}_{2}^{2}\right| \leq \begin{cases}\frac{2 l\left(2 \lambda^{2} \mathrm{t}-\lambda \mathrm{t}+1\right)}{B} ; & \text { for }|\mu-1| \leq \frac{\mathrm{A}}{4 l^{2} B} \\ \frac{8 l^{3}|1-\mu|\left(2 \lambda^{2} \mathrm{t}-\lambda \mathrm{t}+1\right)}{\mathrm{A}} ; & \text { for }|\mu-1| \geq \frac{\mathrm{A}}{4 l^{2} B} .\end{cases}
$$

Proof.

From

and

$$
\begin{aligned}
\mathrm{a}_{3}-\mu \mathrm{a}_{2}^{2}= & (1-\mu) \frac{\mathrm{U}_{1}^{3}(l)\left(2 \lambda^{2} \mathrm{t}-\lambda \mathrm{t}+1\right)^{2}\left(\mathrm{c}_{2}+\mathrm{d}_{2}\right)}{2 \mathrm{U}_{1}^{2}(l)\left[\mathrm{B}\left(2 \lambda^{2}-\lambda \mathrm{t}+1\right)-C\right]-2 U_{2}(l)\left[2-\mathrm{u}_{2}\left(\left(2 \lambda^{2}-3 \lambda+1\right)+2 t\left(2 \lambda^{2}-\lambda\right)\right)\right]^{2}} \\
+\frac{\mathrm{U}_{1}(l)\left(\mathrm{c}_{2}-\mathrm{d}_{2}\right)\left(2 \lambda^{2}-\lambda \mathrm{t}+1\right)}{2 \mathrm{~B}} & \\
= & \mathrm{U}_{1}(l)\left(2 \lambda^{2} \mathrm{t}-\lambda \mathrm{t}+1\right)\left[\left(\mathrm{h}(\mu)+\frac{1}{2 B}\right) \mathrm{c}_{2}+\left(\mathrm{h}(\mu)-\frac{1}{2 B}\right) \mathrm{d}_{2}\right]
\end{aligned}
$$

where

$$
\mathrm{h}(\mu)=\frac{(1-\mu) \mathrm{U}_{1}^{2}(l)\left(2 \lambda^{2} \mathrm{t}-\lambda \mathrm{t}+1\right)}{2 \mathrm{U}_{1}^{2}(l)\left[\mathrm{B}\left(2 \lambda^{2}-\lambda \mathrm{t}+1\right)-C\right]-2 U_{2}(l)\left[2-\mathrm{u}_{2}\left(\left(2 \lambda^{2}-3 \lambda+1\right)+2 t\left(2 \lambda^{2}-\lambda\right)\right)\right]^{2}} .
$$

Then, in view of (1.4), we conclude that

$$
\left|\mathrm{a}_{3}-\mu \mathrm{a}_{2}^{2}\right| \leq \begin{cases}\frac{2 l\left(2 \lambda^{2} \mathrm{t}-\lambda \mathrm{t}+1\right)}{B} & 0 \leq|\mathrm{h}(\mu)| \leq \frac{1}{2 \mathrm{~B}} \\ 4 l|\mathrm{~h}(\mu)| & |\mathrm{h}(\mu)| \geq \frac{1}{2 \mathrm{~B}}\end{cases}
$$

\section{Conclusions}

A modest attempt has been made in this report to study certain class of analytic functions and Sakaguchi type functions on the open unit disk . Introduced new Subclasses of analytic univalent functions in the class $\mathrm{C}_{\Sigma}(\lambda, l, \mathrm{t})$ and by selecting the values $\left(l \in\left(\frac{1}{2}, 1\right], 0 \leq \lambda \leq 1\right.$ and $\left.|\mathrm{t}| \leq 1, \mathrm{t} \neq 1\right)$, we used the Chebyshev polynomial expansions to provided estimates for the initial coefficients of bi-univalent Sakaguchi type functions in $\mathrm{C}_{\Sigma}(\lambda, l, \mathrm{t})$. 


\section{References}

[1] S. Altinkaya and S. Yalcin, "Initial coefficient bounds for a general class of bi-univalent functions", International Journal of Analysis, Article ID 867871 (2014), 4 pp.

[2] S. Altinkaya and S. Yalcin, "Coefficient bounds for a subclass of bi-univalent functions", TWMS Journal of Pure and Applied Mathematics, 6(2) 2015.

[3] D.A. Brannan and T.S. Taha, On some classes of bi-univalent functions, Studia Universitatis BabeșBolyai. Mathematica, 31(2) (1986), 70-77.

[4] D.A. Brannan and J.G. Clunie, "Aspects of comtemporary complex analysis", Proceedings of the NATO Advanced Study Instute Held at University of Durham: july 1-20, 1979). New York: Academic Press, (1980).

[5] S. Bulut, "Faber polynomial coefficient estimates for a comprehensive subclass of analytic bi-univalent functions", C.R. Acad. Sci. Paris, Ser. I, 352 (6) (2014), 479-484.

[6] O. Crișan, "Coefficient estimates certain subclasses of bi-univalent functions”, Gen. Math. Notes, 16 (2) (2013), 93-1002.

[7] P.L. Duren, "Univalent Functions", Grundlehren der Mathematischen Wissenschaften Springer, New York, USA, 2591983.

[8] E.L. Doha, "The first and second kind Chebyshev coefficients of the moments of the general-order derivative of an infinitely differential function," Intern. J. Comput. Math., 51 (1994), 21-35.

[9] S.G. Hamidi and J.M. Jahangiri, "Faber polynomial coefficient estimates for analytic bi-close-to-convex functions," C.R. Acad. Sci. Paris, Ser. I, 352 (1) (2014), 17-20.

[10] J.M. Jahangiri and S.G. Hamidi, "Coefficient estimates for certain classes of bi-univalent functions," Int. J. Math. Sci., Article ID 190560, (2013), 4pp.

[11] M. Lewin, "On a coefficient problem for bi-univalent functions," Proceeding of the American Mathematical Society, 18 (1967), 63-68.

[12] N. Magesh and J. Yamini, "coefficient bounds for a certain subclass of bi-univalent functions," International Mathematical Forum, 8 (27) (2013), 1337-1344.

[13] J.C. Manson, "Chebyshev polynomials approximations for the L-membrane eigenvalue problem," SIAM J. Appl. Math., 15 (1967), 172-186.

[14] E. Netanyahu, "The minimal distance of the image boundary from the orijin and the second coefficient of a univalent function in $|\mathrm{z}|<1$," Archive for Rational Mechanics and Analysis, 32 (1969), 100-112.

[15] S. Porwel and M. Darus, "On a new subclass of bi-univalent functions," J. Egypt. Math. Soc., 21 (3) (2013), 190-193.

[16] B. Srutha keerthi, "Fekete-Szegö Type equalities For certain subclasses of sakaguchi type functions," Romai journal, 8(2), 2012, 119-127.

[17] B. Srutha keerthi and B. Raja, "coefficient inequality for certain new subclasses of analytic bi-univalent functions," Theoretical Mathematics and Applications, 3 (1) (2013), 1-10. 